\title{
A Produção Intelectual Brasileira em Responsabilidade Social Empresarial - RSE sob a Ótica da Bibliometria
}

\section{A Bibliometric Approach to Brazilian Scientific Production on Corporate Social Responsibility - CSR}

Sérgio Luiz do Amaral Moretti * Doutor em Ciências Sociais pela PUC-SP. Professor do PMDA/UNINOVE, São Paulo/SP, Brasil.

Milton de Abreu Campanario

Ph.D. em Economia pela Universidade de Cornell, Estados Unidos. Professor e Coordenador do PMDA/UNINOVE, São Paulo/SP, Brasil.

* Endereço: Sérgio Luiz do Amaral Moretti

Rua Alcantarilla, 87, apto. 121, Morumbi, São Paulo/SP, 05717-170. E-mail: moretti@uninove.br

Copyright @ 2009 RAC. Todos os direitos, inclusive de tradução, são reservados. É permitido citar parte de artigos sem autorização prévia desde que seja identificada a fonte. 


\section{RESUMO}

O presente trabalho visa caracterizar o estado da arte das publicações brasileiras na área da Responsabilidade Social Empresarial [RSE]. Adotou-se o procedimento de traçar o perfil detalhado dos autores, sua produção e citações bibliográficas utilizadas no EnANPAD, entre 1997 e 2007. Além da estatística descritiva sobre autores, obras e citações, foi testada a aplicabilidade da Lei de Lotka - LL às obras citadas. Essa lei afirma que o número de autores (de artigos ou de obras citadas) que fazem $\mathbf{n}$ contribuições em determinado campo científico é aproximadamente $1 / \mathbf{n}^{2}$ daqueles que fazem uma só contribuição. Tal indicador demonstra que, no universo de autores ou de autores citados em um campo científico maduro, há uma distribuição relativamente rígida entre poucos que publicam mais e muitos que publicam menos. Foi possível testar empiricamente que, no âmbito das obras citadas, não há adequação a esta lei, indicando pouca maturidade científica na RSE. A análise mostra também que a produção dos anos anteriores foi pouco utilizada nos anos seguintes e houve repetição de livros textos de administração e autores famosos pouco relacionados ao tema, contribuindo pouco para o avanço científico. Há um domínio da reprodução das mesmas idéias, reforçando a noção de que existe nesta área temática uma zona de conforto intelectual.

Palavras-chave: responsabilidade social empresarial; Lei de Lotka; maturidade científica.

\section{ABSTRACT}

This article develops the selection and analysis of the EnANPAD academic publications between 1997 and 2007. This source of information was object of rigorous and in depth research, including both the profile of the authors, the articles and the quotations. 216 articles were classified with 9,523 corresponding quotations. The quantitative analysis of the quotations aims to describe their characteristics and to test the applicability of the Lotka Law LL, one to the bibliometric fundamentals, which states that the number of authors making $\mathbf{n}$ contributions is about $1 / \mathbf{n} 2$ of those making one contribution. In mature scientific fields, it is expected that few scientists publish more and many publish a less, but under a certain distribution. It was possible to verify that the LL does not apply to the CSR, as it is tested against the quotations used. This means that the field is not mature, in scientific terms. It was also shown that the quotations of one year are not utilized in the following research year. Evidence shows the most frequent quotations are those of administration handbooks and famous authors not necessarily related to CSR. The area is dominated by the reproduction of the same ideas, strengthening the thesis of zone of intellectual comfort.

Key words: corporate social responsibility; Lotka's Law; scientific maturity. 


\section{INTRODUÇÃO}

O campo da Responsabilidade Social Empresarial [RSE], como prática social e como objeto de investigação científica, ainda está em formação no Brasil. O presente estudo busca seguir a tradição acadêmica de saber cada vez mais sobre cada vez menos e aprofundar o conhecimento sobre temas e abordagens cada vez mais específicas, como é o caso da RSE. Este trabalho é baseado em uma pesquisa bibliométrica sobre as publicações acadêmicas desse campo apresentadas no EnANPAD entre 1997 e 2007. Os resultados obtidos permitem uma visualização do perfil dos autores do referido tema, das citações e referências bibliográficas utilizadas e a explicitação de agrupamentos de temas e abordagens mais significativos. Tratando-se de uma área polissêmica, com ampla profusão de significados para os conceitos utilizados - responsabilidade social, sustentabilidade, voluntariado, ética nos negócios e questões ambientais, entre outros - torna-se imprescindível reconhecer as bases de conhecimento sobre as quais o campo se fundamenta (Gomes \& Moretti, 2007). Comparações entre as diversas abordagens sobre RSE poderão fornecer insights para uma discussão mais fundamentada a respeito das direções que o debate tomou nesta última década e suas perspectivas futuras.

Sob essa perspectiva, dois são os objetivos da presente pesquisa. O primeiro é a identificação dos trabalhos e do perfil dos autores por meio do levantamento e da análise de seus Curricula Lattes. Esse procedimento permite reconhecer suas origens, formação, instituições de ensino, áreas de atuação e linhas de pesquisa. O segundo é a classificação e a análise estatística das obras citadas nas referências bibliográficas ou citações utilizadas pelos autores, particularmente com o confronto destas informações com a Lei de Lotka [LL], que é um princípio sobre a produtividade dos autores de um determinado campo científico. Este é considerado o eixo central da pesquisa bibliométrica contemporânea. A geração de novos conhecimentos está diretamente relacionada ao desenvolvimento de novas pesquisas, sendo a regra que uma pesquisa só está terminada quando é publicada ou citada.

Esta Lei da produtividade parte da premissa de que, proporcionalmente, poucos autores publicam mais (e são mais citados) do que muitos autores, que publicam menos. Esta distribuição é verificada em campos avançados da ciência. Conforme Alvarado (2002), esta lei tem a seguinte definição:

Para estabelecer a parte com que homens de diferentes qualidades contribuem ao progresso da ciência, Lotka (1926) estabeleceu os fundamentos da lei do quadrado inverso, afirmando que o número de autores que fazem $\mathrm{n}$ contribuições em um determinado campo científico é aproximadamente $1 / \mathrm{n}^{2}$ daqueles que fazem uma só contribuição. A proporção daqueles que fazem uma única contribuição é de mais ou menos $60 \%$ (p. 14).

Isto significa que, idealmente, $60 \%$ de todos os artigos de uma área são produzidos por um autor. Duas contribuições perfazem cerca de $25 \%$ e três ou mais perfazem cerca de $15 \%$. Esta distribuição será testada contra as citações presentes nos artigos do EnANPAD.

Conforme se observou em estudo anterior (Moretti \& Figueiredo, 2007), a partir de uma amostra de 20 artigos apresentados entre 2003 e 2006 no âmbito da área de Gestão Social e Ambiental [GSA] no EnANPAD, existe a tendência de buscar um referencial dentro da zona de conforto por parte dos autores pesquisados na amostra. Tal fato demonstrou a preocupação de que o discurso da RSE tem um componente reprodutor: referências gerais para facilitar o trânsito dentro do árido campo dos modelos conceituais.

Como qualquer objeto de investigação científica, a RSE não se furta a um debate sobre seu real significado. Friedman (1970) e Jensen (2002), seguindo uma linha liberal, postulam que esse não é um tema empresarial, pois implica ferir a lucratividade; nessa mesma linha, porém argumentando o contrário, Margolis e Walsh (2003) revelam que a conduta socialmente responsável das empresas ou é independente da lucratividade ou a ela está positivamente relacionada. Outros autores pregam o necessário envolvimento da empresa com o tema por razões diversas: a linha estratégica (Carrol, 1999; Ventura, 2003; Wood, 1991) identifica oportunidades de negócios no processo; a da ética dos negócios (Curado, 2003; Ferrel, Fraedrich, \& Ferrel, 2001; Kreitlon, 2004; Lipovetsky, 2004) percebe 
as empresas como agentes portadoras da moral e da ética; a sistêmica ou do stakeholder (Donaldson \& Preston, 1995; Freeman, 2003; Logsdon \& Yuthas, 1997) reconhecem o envolvimento das empresas com os diversos públicos de pressão e interesse. De forma mais pragmática, Gomes e Moretti (2007) apontam que a agenda positiva da sustentabilidade (triple botton line) é uma tendência inexorável do movimento global à qual as empresas devem adaptar-se.

A produção no EnANPAD estabelece novas referências conceituais para a RSE, embora pouco utilizadas na literatura posterior. Curado (2003) trabalha, de forma inédita, o conceito de autoridade, apoiado em informações do Instituto de Pesquisa Econômica Aplicada (2000) e do Instituto Ethos de Empresas e Responsabilidade Social (2001). Ventura (2003) dedica-se a analisar a proposta de Boltanski e Chiapello (2002) sobre o novo espírito do capitalismo e, com o respaldo em Weber (2004), justifica a adequação das empresas aos novos tempos de pressão social. Kreitlon (2004) demonstra que a abordagem da ética de compromisso com a sociedade termina por justificar em si mesma a relevância da RSE, recurso tautológico que requer maior debate.

Outros trabalhos, no entanto, fixaram-se como referências a partir do EnANPAD. Passador, Canopf e Passador (2005) pesquisaram as principais abordagens presentes nesse congresso até 2004, apontando que há um entendimento comum de que a exigência da sociedade é um fator suficiente e justificador (pp. 14-15). O artigo propõe ainda um continuum de classificações para a RSE que varia de modismo a obrigação, passando por legitimidade e ética, o que é bastante útil para inspirar outros pesquisadores. Macke e Carrion (2006) propõem que "seja incluído no debate o conceito interdisciplinar de capital social", justificado pela complexidade dos vetores envolvidos em sua elaboração (p. 15). Sugerindo nova taxonomia para a área, Pereira e Campos (2006) advertem: "as definições acerca da responsabilidade social não demonstram pleno consenso teórico em função do contexto sociocultural e econômico em que cada uma delas foi concebida" (p. 13).

Esse tipo de contribuição presente no EnANPAD nos leva a sugerir a ampliação do debate para a estrutura do sistema e os fundamentos sobre os quais ele está edificado, razão pela qual conduzimos o atual estudo.

Os resultados expostos neste trabalho são exploratórios, embora o EnANPAD seja parte importante da produção intelectual sobre a RSE brasileira. Este trabalho apresenta a bibliometria como disciplina, seguida de apresentação do método de coleta de dados, apresentação e discussão dos resultados, e considerações finais

\section{BIBLIOMETRIA: A CIÊNCIA DAS CIENCIAS}

O aumento da importância da ciência, tecnologia e inovação [C,T\&I] na sociedade fez crescer as métricas sobre a produção intelectual, patentes e informações científicas em geral. $\mathrm{O}$ avanço das tecnologias de informação veio facilitar enormemente o trabalho de mensuração. Entre as abordagens utilizadas, encontram-se a bibliometria, a cienciometria, a informetria e, recentemente, a webmetria. Esses são termos muitas vezes tratados como sinônimos, mas que objetivam atingir, respectivamente, diferentes campos: produção científica, avanços quantitativos da ciência, informação em qualquer formato e conteúdos da Web (Vanti, 2002). Cabe adiantar que atualmente essas métricas são adotadas também na área de negócios. Visando detectar avanços na tecnologia de produtos, processos e serviços, a bibliometria passa a ser incorporada ao campo da Inteligência Competitiva (Boyack, Wylie, \& Davidson, 2002; Rodrigues \& Riccardi, 2007; Tyson, 1998).

As análises da produção científica, das citações e dos conteúdos produzidos constituem objeto da bibliometria (Garfield, 1973; Price, 1965; Zhao, 2006). O termo foi usado pela primeira vez na década de 1920, como conjunto de métodos para medir textos de qualquer natureza, porém só se tornou uma disciplina com o artigo Statistical Bibliography or Bibliometrics?, de Pritchard (1969), que propunha a utilização de métodos quantitativos para investigar a produção escrita como o elemento principal da 
comunicação do conhecimento de C,T\&I. Sobre esse fundamento, a disciplina trata de quantificar a comunicação escrita, utilizando processos variados de levantamento, tratamento e apresentação de dados (Boyack et al., 2002; Guedes \& Borschiver, 2005; Small, 1999). Com a informatização decorrente do avanço tecnológico, a bibliometria conheceu grande impulso, o que lhe permitiu criar mais fácil e precisamente mapas do conhecimento, credenciando-se a tornar-se uma importante ferramenta de políticas em C,T\&I. Um bom exemplo dessa questão é o Manual de Frascati, publicado pela OCDE, que é referência para classificar, medir e conceituar atividades científicas (Bordons \& Zulueta, 1999; Garfield, 1995; Quonian, 1992).

Um mapa do conhecimento é uma representação de como disciplinas, campos de investigação ou produção de indivíduos se inter-relacionam, permitindo estabelecer estruturas e redes de influência e produtividade (White \& McCain, 1989). Diferentes recortes analíticos podem ser utilizados: abordagens, instituições, veículos de publicação, autores, disciplinas etc. O objetivo é sempre mapear e avançar sobre o estado do conhecimento em C,T\&I nos mais diferentes segmentos (Quonian, Tarapanoff, Araújo, \& Alvares, 2001; Small, 1999; Vanti, 2002). O objeto de investigação é a produção científica (artigos, livros), tecnológica (patentes, normas técnicas) ou de inovações (contratos, produtos, processos ou serviços). Essas informações são classificadas e, com o auxílio de softwares de data mining, como Data View (Quonian et al., 2001), Vantagepoint (Amaral et al., 2007), por exemplo, são definidas as matrizes de similaridade, identificadas as redes de influência e realizadas análises de clusters, entre outras técnicas estatísticas. Há também grande avanço nas plataformas de visualização de resultados, como SENTINEL, CAVE e SPIRE, construídas para facilitar a comunicação e a difusão do mapa do conhecimento (Boyack et al., 2002; Small, 1999).

Os estudos bibliométricos podem ser divididos em dois planos: a) o macroplano busca encontrar as unidades básicas estruturais de uma ciência, suas inter-relações e redes, o que ocorre em escala global; b) o microplano concentra a maioria dos mapas de conhecimento e procura estabelecer o melhor conhecimento possível de um domínio disciplinar específico para informar o seu estado da arte (Boyack et al., 2002). Esse segundo plano é o que melhor caracteriza o presente estudo.

Como disciplina científica, a bibliometria desenvolveu um grupo de leis que referenciam os estudos de forma sistemática. A mais conhecida é a Lei de Bradford, que mede o grau de atração de periódicos sobre determinado assunto, mostrando como os artigos se concentram em um núcleo especializado, em torno do qual orbitam os demais periódicos (Guedes \& Borschiver, 2005). Essa lei não se aplica a este trabalho, pois a pesquisa está centrada na produção de uma única fonte (EnANPAD). A Lei de Zipf, por seu turno, mede a frequência de palavras em um determinado texto e é utilizada para estimar que assunto científico ou tecnológico é tratado (Guedes \& Borschiver 2005); por ser uma lei que exige um tratamento muito mais detalhado de dados, ela não será aqui abordada.

A lei que rege a produtividade de autores, como dito acima, é a Lei de Lotka, a terceira e mais importante da bibliometria. Existe uma farta literatura teórica e empírica sobre a Lei de Lotka; contudo, como aponta Nicholls (1989), sua aplicação redunda em conclusões muitas vezes polêmicas devido a formas da medição, parâmetros de estimação, teste e interpretação do modelo proposto por Lotka. Esta lei não se aplica bem a pequenas amostras ou a uma única revista especializada. Mais bem ela é utilizada em grande volume de publicações, em área científica consolidada. Alvarado (2002) afirma que, devido à necessidade de superar estes fatores limitantes, as aplicações mais recentes da Lei de Lotka têm replicado o modelo, usando as citações reportadas na revisão da literatura e não diretamente na autoria dos trabalhos. Exatamente este procedimento é que será utilizado neste trabalho, isto é, o universo de pesquisa são as obras citadas (9.523 citações) para o teste da Lei de Lotka ao invés da autoria (216 artigos) direta na EnANPAD. Por este meio é possível evitar a limitação de trabalhar com um universo pequeno e que poderia distorcer os resultados.

Ademais, para o entendimento da produtividade e do avanço do conhecimento é muito útil verificar quais são os autores e centros de pesquisa mais produtivos e que mais contribuem para o desenvolvimento de um campo de estudo. Quanto mais uma área estiver consolidada, maior é a probabilidade de seus autores produzirem mais artigos dentro de um período de tempo (Guedes \& Borschiver, 2005). Esse procedimento revelou-se extremamente útil para desvelar um campo como o 
da RSE, ainda em desenvolvimento no Brasil. Um pressuposto da pesquisa foi buscar colégios invisíveis, ou seja, um pequeno grupo de autores que são os líderes de certa área e têm seus trabalhos mais citados pelos menos conhecidos (Pao, 1989).

A busca de indicadores norteia todo o processo bibliométrico (Spinak, 1998). Os indicadores são classificados como sendo de uma ou duas dimensões: a) uma dimensão: de atividades (contagem de publicações) e de impacto (contagem das citações), o que é um dos propósitos deste estudo; b) duas dimensões: estabelecem ligações ou elos entre pesquisadores, instituições e países (Amaral et al., 2007; Holbrook, 1992).

Para o caso de C,T\&I, incluindo as Ciências Sociais Aplicadas, pode-se visualizar uma cadeia de indicadores de avaliação que mostra as diversas fases em que uma pesquisa pode ser realizada assim como sua inter-relação. Conforme apontam Mugnaini, Jannuzzi e Quonian (2004), esses indicadores podem ser: insumo (investimentos em $\mathrm{P} \& \mathrm{D}$, pesquisadores e grupos de pesquisa); processo (taxa de titulação de doutores, congressos e feiras científicas); produto (artigos publicados, patentes registradas); impacto (número de citações, patentes, royalties etc). $\mathrm{O}$ indicador de impacto científico mais conhecido é o da Thompson Scientific, por meio da Web of Knowledge do Institute for Scientific Information [ISI]. Trata-se de plataforma virtual que oferece um gerenciador de informações e um acesso único para os periódicos indexados em suas bases: Science Citation Index [SCI], Social Science Citation Index [SSCI] e Arts \& Humanities Citation Index [AHCI], entre os principais (Bordons \& Zulueta, 1999; Institute for Scientific Information [ISI], 2007).

Um bom exemplo da utilidade desses indicadores pode ser encontrado em Stremersch, Vernieers e Verhoef (2007), que pesquisaram todos os artigos de Marketing nos 5 principais periódicos sobre o assunto nos EUA, entre 1990 e 2002. Eles contaram todas as citações de cada artigo, classificando-os segundo a qualidade, a visibilidade e a promoção pessoal do autor. As citações dependem mais do que é dito (qualidade e domínio) e de por quem é dito (visibilidade e promoção pessoal do autor) do que de como é dito (extensão do título, destaques para chamar a atenção, clareza da exposição). Sobre os periódicos de Marketing, o estudo de Baumgartner e Pieters (2003) confirmou a influência do Journal of Marketing e a decisiva influência dessa e outras importantes publicações no estabelecimento do Marketing como disciplina. Leong (1989) e McAlister (2005) desenvolveram estudos semelhantes, o primeiro para o Journal of Marketing e o segundo para a base de dados do Journal of Marketing Research.

Sem o mesmo prestígio que o ISI, outros indicadores são relevantes: a Library and Information Science Abstracts [LISA]; o Education Resource Information Center [ERIC]; a Pascal, rede bibliográfica francesa; o Dialog, publicado pela Thomson Reuters, que disponibliza mais de 800 milhões de informações chave em diversas áreas; o CiteSeer, para o campo das ciências da computação e informação; o RePec, para a área da economia; o Scopus, publicado pela Elsevier para acesso on line para ciências e ciências sociais, e finalmente o Google Scholar, um fácil acesso para citações de anos mais recentes (Alvarado, 2002). A aplicação de técnicas de data mining sobre a produção científica foi bem demonstrada por Quonian et al. (2001), ao estudarem a base de dados de teses francesas Doc Thèses, entre os anos de 1969 e 1999, sobre referências ao Brasil. No plano macro, ainda no caso brasileiro, existem indicadores de C,T\&I publicados pelo Ministério de Ciência e Tecnologia e várias organizações da área. Aqui basta ressaltar o que Mugnaini et al. (2004) atestam: os indicadores na área de ciências sociais aplicadas são praticamente inexistentes se confrontados com a área de C,T\&I, sendo ainda incipiente a pesquisa em bancos de dados como Scielo, Lattes, IBICT, CNPq, CAPES e universidades.

De fato, diretamente ligados ao procedimento metodológico deste trabalho, poucos estudos puderam ser encontrados; a publicação se encontra concentrada na área de Administração e Contabilidade, tendo como destaques os trabalhos de Bertero, Binder e Vasconcelos (2003) sobre estratégia, novamente de Bertero, Caldas e Wood (2005) na organização de um livro sobre a produção científica em administração, de Vieira (2003) sobre a publicação brasileira de Marketing, mesmo assunto a que se dedicaram Caldas, Tinoco e Chu (2003) e Sampaio e Perin (2006) sobre RH. Contudo, sobre exemplos de estudos bibliométricos no Brasil, Alvarado (2002) indica que, nas Ciências Sociais, 
houve somente a participação de 2 estudos (Direito e Marketing) contra 9 estudos de outras áreas que aplicaram a LL. Os demais trabalhos sobre indicadores versavam sobre a área de C,T\&I ou a ciência da bibliometria que, para nós, foi útil como reforço ao procedimento metodológico adotado.

Deve-se ressaltar também uma séria limitação à utilização de indicadores internacionais, devido ao grande número de revistas científicas não indexadas em países em desenvolvimento (Mugnaini et al., 2004). Outras limitações a serem consideradas são o desafio de escapar de abordagens exclusivamente quantitativas e buscar avaliar a qualidade dos trabalhos e as diferenças entre as áreas (Bordons \& Zulueta, 1999).

Thompson (2002), em artigo seminal, demonstrou que as diferenças de forma de publicação entre as áreas são significativas. De fato, 91,3\% das citações em Biologia Molecular provinham de artigos de periódicos e $4 \%$ de monografias (livros), enquanto em humanidades $75 \%$ vinham de monografias e $20 \%$ de periódicos. Há grande incidência de estudos individuais em detrimento de coautoria em humanidades, o inverso ocorrendo nas ciências exatas, da terra e biológicas. Essas evidências demandam aprofundamentos nos estudos de publicações nas áreas sociais (Thompson, 2002). É o que este artigo se propõe a fazer no campo da RSE.

\section{METODOLOGIA}

O presente trabalho se baseou em pesquisa exploratória de caráter quantitativo descritivo. Sem contar com os recursos da ISI Web of Knowledge, procedeu-se à identificação dos dados necessários por meio de trabalho rigoroso no banco de dados do EnANPAD do período de 1997 a 2007 . As grandes etapas metodológicas foram:

1. Selecionar os artigos que tratavam da RSE no EnANPAD. Até 2003 não havia sido criada a área de Gestão Social e Ambiental, GSA, nem a sua sucedânea Administração Pública e Gestão Social, APS. Essa fase foi resolvida pela leitura de todos os títulos e resumos dos artigos de cada ano do evento, assim como seus resumos, para identificar sua adesão ao tema pesquisado.

2. Depois de 2003 ainda se tornou necessário proceder da mesma forma, devido à elasticidade com que o tema é tratado, podendo estar localizado em qualquer das áreas temáticas do congresso, o que se verificou ser verdadeiro.

3. Os artigos com seu texto integral foram arquivados pelo ano de publicação para posterior separação por grupos temáticos específicos dentro do tema RSE. Os critérios trabalhados no projeto preliminar apresentado no EnANPAD de 2007 (Moretti \& Figueiredo, 2007) serviram de base para essa classificação inicial.

4. Foram detectados cinco grupos principais nos 329 artigos classificados para a pesquisa, conforme se demonstra na Tabela 1. O Terceiro Setor foi excluído, pois o tema principal da pesquisa é a responsabilidade social praticada pelas empresas e não por entidades desse setor, normalmente executoras e não patrocinadoras de ações sociais. Foram considerados, portanto, os 216 artigos classificados nos demais grupos. 
Tabela 1: Grupos temáticos sobre RSE no EnANPAD entre 1997 e 2007

\begin{tabular}{l|l|l|l|l|l|l|l|l|l|l|l|l}
\hline & $\mathbf{9 7}$ & $\mathbf{9 8}$ & $\mathbf{9 9}$ & $\mathbf{0 0}$ & $\mathbf{0 1}$ & $\mathbf{0 2}$ & $\mathbf{0 3}$ & $\mathbf{0 4}$ & $\mathbf{0 5}$ & $\mathbf{0 6}$ & $\mathbf{0 7}$ & Total \\
\hline Terceiro Setor & 2 & 4 & 3 & 4 & 7 & 9 & 8 & 21 & 18 & 19 & 18 & 113 \\
Gestão Social & 0 & 0 & 0 & 1 & 0 & 5 & 6 & 3 & 6 & 7 & 3 & 31 \\
Marketing & 1 & 1 & 1 & 2 & 2 & 3 & 7 & 8 & 4 & 8 & 10 & 47 \\
Fundamentos & 1 & 0 & 1 & 2 & 5 & 5 & 7 & 14 & 16 & 12 & 17 & 80 \\
Gestão Ambiental & 2 & 0 & 0 & 1 & 2 & 0 & 8 & 19 & 8 & 13 & 5 & 58 \\
Subtotal RSE & 4 & 1 & 2 & 6 & 9 & 13 & 28 & 44 & 34 & 40 & 35 & 216 \\
Artigos RSE + TS & 6 & 5 & 5 & 10 & 16 & 22 & 36 & 65 & 52 & 59 & 53 & 329 \\
\hline
\end{tabular}

Fonte: elaborado pelos autores.

5. Cada artigo foi analisado para identificar o foco a que se propunha e organizado segundo os temas com as seguintes premissas. a) Fundamentos: razões da adoção das práticas de RSE, ética e cidadania. b) Marketing: ações de comunicação, balanço social, reações dos consumidores. c) Gestão Social: atividades praticadas pelas empresas, relatos de casos práticos, gestão interna. d) Gestão Ambiental: atividades das empresas e ambientes construído e natural, casos práticos das atividades das empresas.

6. O primeiro objetivo da pesquisa foi traçar o perfil dos autores que publicaram no EnANPAD, no período. Seus dados foram tratados mediante o levantamento das informações no currículo Lattes. Os perfis foram arquivados em Excel e manipulados de forma a mostrar os indicadores por cada um dos grupos e pelo total.

7. O segundo objetivo, a constituição de um banco de dados sobre as obras citadas pelos autores dos artigos, foi muito mais trabalhoso, devido à ausência de qualquer estudo anterior - a não ser o de Moretti e Figueiredo (2007). Os dados foram colhidos um a um. Os nomes de cada uma das obras citadas (referências bibliográficas) foram arquivados em Excel e, pelo seu sistema de filtro, procedeu-se ao somatório por grupo e pelo total. Os resultados foram tratados estatisticamente para fornecer os gráficos e tabelas que constam da seção seguinte.

É importante lembrar que a pesquisa, na falta de um software que pudesse identificar as citações no corpo dos artigos, focou sua busca nas obras citadas nas referências bibliográficas constantes do final de cada artigo. Esse procedimento cobre todas as referências incluídas nos textos; contudo não chega a especificá-las em detalhes. Lembramos que os indicadores SSCI da ISI não cobrem periódicos nacionais. Dessa forma, o segundo objetivo teve de ser resolvido mediante o manuseio direto, tendo sido o que levou o maior tempo para ser processado. O processamento estatístico utilizou o software SPSS 15.0.

Pelo fato de existirem 2 diferentes tipos de autores a serem considerados nos resultados, adotou-se a denominação autores para aqueles que produziram os artigos pesquisados na EnANPAD e a denominação obras citadas ou citações para os trabalhos e seus autores citados na referência bibliográfica. Os primeiros serão objeto de estudo de perfil; os segundos, de estudo bibliométrico, como na análise de resultados será mostrado.

\section{RESULTADOS E DISCUSSÃO}

\section{Perfil dos Autores}

O primeiro objetivo foi traçar o perfil dos autores por meio do currículo Lattes. Como esperado, por se tratar de um congresso de Administração, a maioria se encontra dentro da grande área das Ciências Sociais Aplicadas, compreendendo 292 autores ou 71,2\% do total de 410. Na área de Administração 
estão $65,4 \%$ e os restantes $34,6 \%$ encontram-se em outras áreas. Não sendo pequena a participação de outras áreas abre-se a possibilidade de futuros estudos investigarem a interdisciplinaridade que se forma na RSE.

\section{Linhas de Pesquisa}

Em relação às linhas de pesquisa, 180 dos 410 autores não declararam nenhuma opção, nos restantes identificaram-se 103 linhas de pesquisas diversas. Adotou-se o critério de registrar a primeira marcação, por ser a mais importante e considerar somente o total de 75 linhas $(72,8 \%)$ que mantinham alguma relação com os critérios de cada grupo. Interessante é notar que $27,2 \%$ se dedicam a outras linhas de pesquisa não relacionadas aos critérios dos grupos, sugerindo um processo que merece ser investigado em outra pesquisa. Dessa forma obteve-se a seguinte distribuição das linhas de pesquisa: Fundamentos $=21$; Marketing $=16$; Gestão Social $=7$ e Gestão Ambiental $=37$, sendo o grupo com maior diferenciação entre as linhas.

\section{Gênero e Titulação dos Autores dos Artigos Pesquisados}

O perfil por gênero dos autores e coautores dos artigos dentro do universo pesquisado foi retirado de seus curricula Lattes e mostra o equilíbrio entre homens e mulheres, em uma área que já foi dominada pelos homens, conforme a Tabela 2. Esse resultado sugere não simplesmente um ciclo, mas uma tendência que poderá ser comprovada nos próximos anos. A questão fica mais clara, observando-se a evolução da participação de cada um deles ao longo dos anos. O período foi dividido em quartis por ano de formação dos autores, considerando-se a seguinte datação: $1^{\circ}-1970-1987,2^{\circ}-1988-1994,3^{\circ}$ - 1995-2001 e $4^{\circ}-2002-2007$.

Tabela 2: Distribuição dos Autores e Coautores por Gênero e Período de Titulação

\begin{tabular}{l|l|l|l|l|l|l|l|l|l|l|l|l}
\hline & $\mathbf{1}^{\mathbf{0}}$ & $\mathbf{\%}$ & $\mathbf{2}^{\mathbf{0}} \mathbf{Q}$ & $\mathbf{\%}$ & $\mathbf{3}^{\mathbf{0}}$ & $\mathbf{\%}$ & $\mathbf{4}^{\mathbf{0}} \mathbf{Q}$ & $\mathbf{\%}$ & $\mathbf{N I}$ & $\mathbf{\%}$ & Total & $\mathbf{\%}$ \\
\hline Homens & 10 & 71 & 19 & 83 & 47 & 54 & 120 & 48 & 17 & 47 & 213 & 52 \\
Mulheres & 4 & 29 & 4 & 17 & 39 & 46 & 131 & 52 & 19 & 53 & 197 & 48 \\
Total & 14 & & 23 & & 86 & & 251 & & 36 & & 410 & 100 \\
\% & 3,4 & & 5,6 & & 21 & & 61 & & 9 & & 100 \\
\hline
\end{tabular}

(NI: Não informaram).

Fonte: elaborado pelos autores.

O ano da última titulação dos autores e coautores indica que o processo de renovação na área do EnANPAD é bastante alto, com cerca de 70\% deles se titulando no último quartil (2002-2007) e 90\% nos dois últimos. Esse resultado, combinado com o exposto na tabela anterior, parece demonstrar que existe a produção de trabalhos acadêmicos frutos de orientações de mestrado e doutorado. Ademais, a Tabela 3 mostra maior presença de doutores, indicando uma evolução no número e na formação de pesquisadores na área de RSE. A distribuição semelhante da titulação pelos grupos permite inferir a validade dos critérios de classificação que foram adotados para eles.

Tabela 3: Perfil dos Autores e Coautores: Titulação por Grupo e Total

\begin{tabular}{l|l|l|l|l|l|l}
\hline & Fundamentos & Marketing & G. Social & G. Ambiental & Total & \% s/ Total \\
\hline Livre- Docência & 0 & 5 & 2 & 3 & 10 & 2,5 \\
\hline Pós-Doutorado & 11 & 7 & 4 & 11 & 33 & 8,0 \\
\hline Doutorado & 72 & 51 & 29 & 60 & 212 & 51,7 \\
\hline Mestrado & 23 & 40 & 17 & 24 & 104 & 25,4 \\
\hline Outros & 18 & 7 & 9 & 17 & 51 & 12,4 \\
\hline Total & 124 & 110 & 61 & 115 & 410 & \\
\hline
\end{tabular}

Fonte: elaborado pelos autores. 


\section{Autoria e Coautoria}

O número de autores por artigo apresentou resultados aparentemente normais, conforme a Tabela 4. Há uma concentração de coautoria apresentando o agrupamento de dois autores $(45,3 \%)$ e de três autores $(21,7 \%)$. Esse resultado reforça o argumento anterior de que muitas parcerias de orientações continuam sendo importantes geradores de produção acadêmica. Embora não tenha sido tabulado por falta de espaço, o grupo Fundamentos concentrou com 13 autores o maior número dos que apresentaram trabalhos mais de uma vez, seguido pelo grupo Gestão Ambiental, com 6 autores. A diferença entre o número de autores na tabela anterior (410) e o total final averiguado (470) se explica pelas 60 repetições de alguns autores ao longo do período pesquisado.

Tabela 4: Número de Autores por Artigo

\begin{tabular}{l|l|l|l|l|l|l|l}
\hline & 1 & 2 & 3 & 4 & 5 & 6 & Total \\
\hline Artigos & 52 & 98 & 47 & 15 & 3 & 1 & 216 \\
\% & 24,1 & 45,3 & 21,7 & 6,9 & 1,9 & 0,1 & 100 \\
Autores & 52 & 196 & 141 & 60 & 15 & 6 & $470(2,17)$ \\
$\%$ & 11,1 & 41,7 & 30 & 12,8 & 3,2 & 1,2 & 100 \\
\hline
\end{tabular}

Fonte: elaborado pelos autores.

\section{Mapeamento Geral das Obras Citadas}

O segundo objetivo foi mapear as obras citadas pelos autores nas referências bibliográficas; alguns resultados gerais são dignos de nota. A contagem mostrou que, para o total dos quatro grupos, foram 97 as obras citadas em número superior ou igual a 7 vezes. O número de obras citadas nesse mesmo intervalo alcançou 1.247. Lembramos que há repetição dos autores. Essas obras foram originadas de 54 fontes nacionais e 43 estrangeiras. Entre os objetos das obras citadas destacam-se obras relacionadas com a RSE (cerca de 60\%), seguidas pelas obras de Teoria Organizacional (20\%) e Marketing/Negócios (13\%). Um importante ponto de discussão se abre com esse fato: os pesquisadores dos anos mais recentes utilizaram pouco as pesquisas dos anos passados. Seria falta de interesse por elas por parte dos novos pesquisadores? Ou seria a ausência de uma revisão mais rigorosa da literatura? Essas questões ficam abertas para novos estudos.

A distribuição da origem das citações apresenta uma relação à primeira vista não adequada; os artigos e o material de congressos obtêm $46 \%$ das menções, os livros somam $43 \%$. Os artigos trazem as maiores novidades para a pesquisa. A frequência de livros relativamente alta parece indicar uma estratégica adesão à zona de conforto, em prejuízo das novas propostas típicas dos artigos.

\section{Obras Citadas com até 18 Citações}

Os dados da Tabela a seguir chamam a atenção para a extrema dispersão das obras citadas pelos autores dos 216 artigos. Se levarmos em conta que se trata de um estudo longitudinal com uma cobertura de 11 anos e que foram identificadas 9.523 obras, percebe-se que poucos autores dos anos anteriores foram utilizados nos anos seguintes. Esta observação origina-se na constatação de que o alto índice de obras citadas até 10 vezes $(88 \%)$ revela que poucas obras foram citados muitas vezes (1 delas, 54 vezes, outra 52 vezes e mais uma 49 vezes, ver Tabela 6), demonstrando concentração das citações em poucos autores. 
Tabela 5: Número de Citações das Obras Utilizadas pelos Autores dos Artigos

\begin{tabular}{l|l|l|l}
\hline $\begin{array}{l}\text { Número de citações } \\
\text { das obras }\end{array}$ & $\begin{array}{l}\text { Número de vezes que } \\
\text { ocorreram citações }\end{array}$ & Total de citações & $\%$ \\
\hline & & & \\
1 & 4158 & 4158 & 43,6 \\
2 & 824 & 1648 & 17,3 \\
3 & 317 & 951 & 9,9 \\
4 & 139 & 656 & 5,8 \\
5 & 122 & 282 & 6,4 \\
6 & 47 & 168 & 2,9 \\
7 & 24 & 152 & 1,7 \\
8 & 19 & 72 & 0,16 \\
9 & 8 & 90 & 0,076 \\
10 & 9 & 8.687 & 0,095 \\
Total até 10 citações & & 9.523 & 88,0 \\
Até 40 & &
\end{tabular}

Fonte: elaborado pelos autores.

A Tabela 6 mostra a quantidade de obras que obtiveram mais de 18 citações, limitada a esse número pelo espaço disponível; outras tabelas mostrarão as relações estatísticas para as quantidades totais averiguadas. Pode-se observar o domínio de citações para as obras de abordagem geral, como é o caso do Handbook de Estudos Organizacionais, cujos três volumes foram lançados em anos diferentes no Brasil, que é a tradução da edição inglesa de 1996. O total de 88 citações para essa referência revela que o suporte bibliográfico para aspectos gerais é assimétrico em relação a obras específicas de RSE. Deve-se observar que somente um capítulo dos três volumes da edição brasileira trata da temática ambiental e, surpreendentemente, não foi apontado por nenhum dos autores.

Tabela 6: Obras Citadas por Grupo e Total: até 18 Citações

\begin{tabular}{|c|c|c|c|c|c|}
\hline Referências bibliográficas & Fund & Mkt & G.I & G.A & Total \\
\hline $\begin{array}{l}\text { Ashley, P. A. (2001). Ética e responsabilidade social nos negócios. São } \\
\text { Paulo: Saraiva. }\end{array}$ & 40 & 14 & & & 54 \\
\hline $\begin{array}{l}\text { Instituto Ethos de Empresas e Responsabilidade Social. (2002). Guia de } \\
\text { elaboração do balanço social-2002. Recuperado em } 22 \text { março, 2003, de } \\
\text { http://www1.ethos.org.br/EthosWeb/pt/307/o_instituto_ethos/o_que_fazemos/ferra } \\
\text { mentas_de_gestao/guia_de_balanco_social/guia_de_balanco_social.aspx }\end{array}$ & 19 & 22 & 21 & & 52 \\
\hline $\begin{array}{l}\text { Melo, F. P., Neto, \& Froes, C. (1999). Responsabilidade social e } \\
\text { cidadania empresarial. Rio de Janeiro: Qualitymark. }\end{array}$ & 19 & 15 & 15 & & 49 \\
\hline $\begin{array}{l}\text { Clegg, S. R., Hardy, C., Nord, W. (Eds.). (1996). Handbook of } \\
\text { organization Studies [Introduction: organizations, organization and } \\
\text { organizing]. London: Sage. }\end{array}$ & 33 & & & & 33 \\
\hline $\begin{array}{l}\text { Hardy, C. (1998). Handbook de estudos organizacionais: modelos de } \\
\text { análise e novas questões em estudos organizacionais [Introdução: } \\
\text { organização e estudos organizacionais]. São Paulo: Atlas. }\end{array}$ & 30 & & & & 30 \\
\hline $\begin{array}{l}\text { Freeman, R. E. (1999). Stakeholders, social responsibility, and } \\
\text { performance: empirical evidence and theoretical perspectives. Academy } \\
\text { of Management Journal, 42(5), 479-485. }\end{array}$ & 28 & & & & 28 \\
\hline $\begin{array}{l}\text { Porter, M. E. (1995). Green and competitive: ending the stalemate. } \\
\text { Harvard Business Review, 73(5), 120-134. }\end{array}$ & 27 & & & & 27 \\
\hline $\begin{array}{l}\text { Fischer, T. (1998). Handbook de estudos organizacionais: modelos de } \\
\text { análise e novas questões em estudos organizacionais (Vol. 1). São } \\
\text { Paulo: Atlas. }\end{array}$ & 17 & & 8 & & 25 \\
\hline $\begin{array}{l}\text { Silva, C. A. T., \& Freire, F. S. (Orgs.) (2001). Balanço social: teoria e } \\
\text { prática. São Paulo: Atlas. }\end{array}$ & & 24 & & & 24 \\
\hline
\end{tabular}




\section{(conclusão)}

\section{Tabela 6: Obras Citadas por Grupo e Total: até 18 Citações}

\begin{tabular}{|c|c|c|c|c|c|}
\hline Referências bibliográficas & Fund & Mkt & G. I & G.A & Total \\
\hline $\begin{array}{l}\text { Vieira, M. M. F. (1999, setembro). Contribuições da perspectiva } \\
\text { institucional para a análise das organizações. Anais do Encontro } \\
\text { Nacional da Associação Nacional de Pós-Graduação e Pesquisa em } \\
\text { Administração, Foz do Iguaçu, PR, Brasil, } 23 \text {. }\end{array}$ & 23 & & & & 23 \\
\hline $\begin{array}{l}\text { Carroll, A. B. (1979). A three-dimensional conceptual model of } \\
\text { corporate performance. Academy of Management Review,4(4), 497-505. }\end{array}$ & 23 & & & & 23 \\
\hline $\begin{array}{l}\text { Kroetz, C. E. S. (2001). Balanço social: uma proposta de normatização. } \\
\text { Revista Brasileira de Contabilidade, (129), 53-63. }\end{array}$ & & 23 & & & 23 \\
\hline $\begin{array}{l}\text { Kotler, P. (1978). Marketing para organizações que não visam lucro } \\
\text { (H. Barros, Trad.). São Paulo: Atlas. (Obra original publicada em } \\
\text { 1975). }\end{array}$ & & 23 & & & 23 \\
\hline $\begin{array}{l}\text { Ventura, E. C. F. (1999). Responsabilidade social das organizações: } \\
\text { estudo de caso no Banco Central do Brasil. Dissertação de mestrado, } \\
\text { Fundação Getúlio Vargas, Rio de Janeiro, RJ, Brasil. }\end{array}$ & 19 & & & & 19 \\
\hline $\begin{array}{l}\text { Mintzberg, H. (2000). Safári da estratégia: um roteiro pela selva do } \\
\text { planejamento estratégico. Porto Alegre: Bookman. }\end{array}$ & 19 & & & & 19 \\
\hline $\begin{array}{l}\text { Friedman, M. (1970, September 13). The social responsibility of } \\
\text { business is to increase its profits. New York Times Magazine. }\end{array}$ & 18 & & & & 18 \\
\hline
\end{tabular}

Fonte: elaborado pelos autores.

Se às menções ao Handbook adicionarmos 19 referências para Mintzberg, que notoriamente trata de estratégia, já teremos um total de 107 citações para obras que não estão ligadas diretamente ao tema da RSE. Este montante representa $24 \%$ do total das obras citadas em detrimento daquelas mais diretamente focadas no tema da RSE e, em especial, nos 4 grupos estudados. No entanto devemos considerar que o campo da RSE é favorável aos estudos interdisciplinares, nos quais o diálogo entre áreas diferentes de conhecimento se torna fundamental. Esse é um objeto importante de futuras pesquisas.

\section{Teste Estatístico da Lei de Lotka [LL] para as Citações}

Visando obter indicadores mais consistentes sobre os padrões das citações, foram utilizados testes estatísticos para verificar a aplicação da Lei de Lotka [LL] no universo das obras citadas. Utilizou-se o método de aderência com o cálculo do coeficiente $\mathrm{C}$ da referida lei. $\mathrm{O}$ coeficiente $\mathrm{C}$ é estimado segundo a amostra e o seu padrão na LL é igual a 2. Valores acima desse coeficiente indicam que autores com uma publicação são maiores, em termos percentuais, do que o padrão da LL.

Pudemos verificar que não se confirmou a relação. A base das citações com 1 ou mesmo 2 autores é mais que proporcional aos indicadores da LL, indicando que esses autores são pouco utilizados nas pesquisas seguintes. Existe de fato uma concentração - como a tabela anterior demonstrou - em poucos autores de teoria geral da administração ou de estratégia. Essa é uma indicação de que a forma como os trabalhos são desenvolvidos contribui reduzidamente para o avanço desse campo, por terem suas idéias já assimiladas e difundidas. Há um domínio da reprodução das mesmas idéias, reforçando a tese de zona de conforto intelectual.

Uma das críticas do trabalho de Alvarado (2002) aos estudos bibliométricos realizados no Brasil é que não utilizaram testes estatísticos para avaliar o ajustamento dos dados empíricos de produtividade. Visando minimizar essa deficiência, optou-se por utilizar o teste Kolmogorov-Smirnov [K-S], para avaliar o ajuste da distribuição teórica da LL com a distribuição observada sobre as obras citadas em cada um dos quatro grupos analisados. Segundo Siegel (1975), o teste K-S é uma prova de aderência, uma vez que se refere ao grau de concordância entre a distribuição de um conjunto de dados amostrais 
(observados) e uma distribuição teórica específica. É mais uma medida do ajuste de uma distribuição de frequências similar ao $\chi^{2}$ (qui-quadrado). Não obstante, o K-S é mais poderoso e mais fácil de usar que o $\chi^{2}$ (qui-quadrado), não sendo necessário que os dados estejam agrupados em frequências inferiores a 5 como este último exige. O K-S é particularmente útil para julgar o grau de proximidade das frequências observadas em face das frequências esperadas. Foram estabelecidas duas hipóteses para o teste:

H0: Não existe diferença significativa entre a distribuição observada e a LL, ou seja, a distribuição se ajusta à LL.

H1: Existe diferença significativa entre a distribuição observada e a LL, ou seja, a distribuição não se ajusta à LL.

Para efetuar o teste, trabalhou-se com um nível de significância de 0,01, por meio do software estatístico SPSS 15.0. Os resultados bem como a análise descritiva são apresentados nas duas tabelas a seguir. A Tabela 7 mostra a estatística descritiva para as variáveis na análise. Essa estatística oferece descrições de medidas centrais, variabilidade, assim como valores mínimos e máximos das variáveis. Essa estatística nos ajuda a indicar quais técnicas não paramétricas devem ser empregadas em vez dos métodos paramétricos.

Tabela 7: Análise Descritiva

\begin{tabular}{l|llllll}
\hline Grupos & & N & Média & $\begin{array}{l}\text { Desvio } \\
\text { Padrão }\end{array}$ & Mínimo & Máximo \\
\hline Fundamentos & Total Geral & 2.007 & 1,89 & 2,557 & 1 & 40 \\
& Padrão LL & 2.012 & 0,16 & 0,364 & 0 & 1 \\
Marketing & Total Geral & 1.285 & 1,62 & 1,977 & 1 & 24 \\
G. Social & Padrão LL & 1.286 & 0,11 & 0,314 & 0 & 1 \\
& Total Geral & 890 & 1,59 & 1,608 & 1 & 21 \\
G. Ambiental & Padrão LL & 891 & 0,11 & 0,317 & 0 & 1 \\
& Total Geral & 1.343 & 1,41 & 1,009 & 1 & 17 \\
& Padrão LL & 1.344 & 0,10 & 0,295 & 0 & 1 \\
\hline
\end{tabular}

Fonte: elaborado pelos autores.

Como todas as variáveis estão na mesma escala de medida, analisando diretamente as médias se pode comparar os quatro grupos, a fim de identificar padrões nos dados. Observamos que o grupo Fundamentos obteve a maior média entre os grupos, com o valor médio de 1,89 citações, e também o maior desvio padrão entre os grupos: 2,557. Técnicas não paramétricas foram empregadas no estudo, devido às discrepâncias dos valores do desvio padrão. 
Tabela 8: Total dos Grupos, Frequência com 2 Amostras e Valores por Grupo

\begin{tabular}{|c|c|c|c|c|c|c|c|}
\hline Grupos & Autores base LL & $\mathbf{N}$ & $\mathbf{C}$ & $\mathbf{K S}$ & P Value & Alfa & Decisão \\
\hline \multirow[t]{3}{*}{ Fundamentos } & Com 1citação e menores que LL & 1.691 & \multirow{3}{*}{2} & \multirow{3}{*}{16,317} & \multirow{3}{*}{0,000} & \multirow{3}{*}{0,01} & \multirow{3}{*}{$\begin{array}{l}\text { Rejeito H0 - } \\
\text { são diferentes }\end{array}$} \\
\hline & Com1citação e maiores que LL & 316 & & & & & \\
\hline & Total & 2.007 & & & & & \\
\hline \multirow[t]{3}{*}{ Marketing } & Com 1citação e menores que LL & 1144 & \multirow{3}{*}{2} & \multirow{3}{*}{11,239} & \multirow{3}{*}{0,000} & \multirow{3}{*}{0,01} & \multirow{3}{*}{$\begin{array}{l}\text { Rejeito H0 - } \\
\text { são diferentes }\end{array}$} \\
\hline & Com 1 citacão e maiores que LL & 142 & & & & & \\
\hline & Total & 1.286 & & & & & \\
\hline \multirow[t]{3}{*}{ Gestão Social } & Com 1 citação e menores que LL & $\begin{array}{l}789 \\
101\end{array}$ & \multirow{3}{*}{2} & \multirow{3}{*}{9,462} & \multirow{3}{*}{0,000} & \multirow{3}{*}{0,01} & \multirow{3}{*}{$\begin{array}{l}\text { Rejeito H0 - } \\
\text { são diferentes }\end{array}$} \\
\hline & Com1 citação e maiores que LL & 890 & & & & & \\
\hline & Total & & & & & & \\
\hline \multirow{3}{*}{$\begin{array}{l}\text { Gestão } \\
\text { Ambiental }\end{array}$} & Com1 citação e menores que LL & 1.214 & \multirow{3}{*}{2} & \multirow{3}{*}{10,799} & \multirow{3}{*}{0,000} & \multirow{3}{*}{0,01} & \multirow{3}{*}{$\begin{array}{l}\text { Rejeito H0 - } \\
\text { são diferentes }\end{array}$} \\
\hline & Com 1 citação e maiores que LL & 129 & & & & & \\
\hline & Total & 1.343 & & & & & \\
\hline
\end{tabular}

*Diferenças extremas - absoluta: 1,000 indica 100\%; significância alta $\geq 0,01$ indica aderência à LL.

Fonte: elaborado pelos autores.

\section{Interpretação do Ajuste da Distribuição as Citações à Lei de Lotka}

Já que são apenas duas alternativas, existem somente duas formas de interpretação: a distribuição se ajusta à Lei de Lotka ou a distribuição não se ajusta à Lei de Lotka.

a) Se Asymp. Sig (2-tailed) é menor que o valor crítico - isto é, o valor crítico é maior que Asymp. Sig (2-tailed) - rejeitamos a hipótese nula de homogeneidade da distribuição de frequências dos autores, ou seja, rejeitamos a hipótese de que essa distribuição se ajusta à Lei de Lotka, ao 0,01 nível de significância.

b) b) Se Asymp. Sig (2-tailed) é maior que o valor crítico - isto é, o valor crítico é menor que Asymp. Sig (2-tailed) - aceitamos a hipótese nula de homogeneidade da distribuição de frequências dos autores, ou seja, aceita-se a hipótese de que essa distribuição se ajusta à Lei de Lotka, ao 0,01 nível de significância.

Como, no caso, todos os valores críticos Asymp. Sig (2-tailed) são menores que o nível de significância adotado $\mathrm{a}=0,01$, então essas distribuições não se ajustam à Lei de Lotka, a um 0,01 nível de significância.

\section{CONSIDERAÇÕES FINAIS}

Os resultados apresentados alcançam a contento os objetivos de mapear e analisar o perfil dos autores de RSE e das obras citadas por eles em suas referências bibliográficas. A metodologia de pesquisa bibliométrica utilizada demonstrou ser de grande valia para tratar a área de RSE, assim como parece ser indicada para outras áreas de pesquisa. Algumas descobertas do estudo anterior de Moretti e Figueiredo (2007) foram confirmadas. Existe exagerada concentração das citações em poucas obras genéricas da área de administração, o que inibe revelar novos talentos e propostas para o campo. $\mathrm{O}$ fato de muitos autores virem sendo introduzidos dentro de um longo período de onze anos e não serem utilizados nos trabalhos posteriores parece significar uma acomodação, da mesma forma que recorrer a expedientes confortáveis e tradicionais de referências de obras de caráter geral torna inócuo o marco 
teórico e impede o aprofundamento temático. Isso implica o reconhecimento de que o campo da RSE está longe de contar com referencial teórico consistente, sugerindo a necessidade de uma agenda de pesquisas mais ambiciosa. Sem evolução nesse quesito que possibilite sustentar o desenvolvimento conceitual da RSE, devido principalmente a sua natureza de se valer de linhas epistemológicas diversas, o campo da RSE ficará dependente de um discurso monológico e pouco autêntico.

A busca de referencial dentro da zona de conforto por parte dos autores pesquisados demonstrou que o discurso da RSE tem forte componente reprodutor. Observou-se que os autores lançam mão de referências gerais e de outras disciplinas para transitar dentro do campo conceitual específico. Tomese o caso de Ashley (2003) e Melo e Froes (2001), com obras relevantes para abordar vários aspectos da RSE em um momento de sua solidificação como campo de estudo. Ambas apresentam esquemas interpretativos e introduzem a bibliografia estrangeira relevante sobre o tema. Não deixa de ser interessante que todos os estrangeiros do quadro de maiores frequências estão presentes nas 2 obras, o que parece indicar que, nesse caso, fizeram escola; mas, na sequiência, não houve nenhuma tentativa de introduzir autores mais recentes. Como dissemos, estamos diante da síndrome da zona de conforto intelectual nesse campo.

Apontamos na seção Resultados e discussão algumas propostas para novos estudos. Para o mapeamento de autores, abre-se a possibilidade de estudos investigarem a interdisciplinaridade que se forma na RSE, a participação de autores de outras linhas e ainda a distribuição por gênero. A origem de formação e vínculos institucionais por grupos de pesquisa podem ser detalhados.

Para o mapeamento das citações, é relevante levar o tratamento estatístico para conferir a LL dentro de um universo maior de artigos, periódicos, teses e dissertações. O EnANPAD é uma boa fonte, mas representa a primeira aproximação de alto nível da produção intelectual junto à comunidade acadêmica. O que pode significar a pouca presença dos artigos sobre a RSE de anos passados em anos recentes? Será que essa evidência se repete nos artigos em revistas, nas teses e dissertações? Seria a ausência de uma revisão mais rigorosa da literatura? Essas questões ficam abertas para novos estudos.

As definições e proposições sobre a RSE variam conforme o contexto em que são formuladas. À medida que novos horizontes de atuação das empresas são alcançados, observa-se a flexibilização das abordagens do papel das empresas na sociedade. $\mathrm{O}$ mesmo pode-se dizer das pressões, à medida que se agravam as desigualdades e a agressão ao meio físico. Essas são algumas das questões que propomos venham a ser discutidas com mais frequência pela comunidade acadêmica.

Este trabalho pode contribuir para os levantamentos de outros campos de estudo, partindo da observação de alguns passos.

a) Deve-se priorizar a busca por referências bibliográficas em periódicos, congressos e teses e dissertações que mantêm a pesquisa atualizada e não em livros cujos resultados já estão sedimentados e se referem a pesquisas passadas. Sem esse procedimento, corre-se o risco de fornecer mais sobre o mesmo.

b) A revisão da literatura deve buscar a atualização de publicações internacionais dos últimos 3 anos, pelo menos. Não foi isso que se viu nas obras citadas nas referências bibliográficas do campo da RSE no EnANPAD. A qualidade da revisão da literatura de qualquer campo deveria ser objeto de mais estudos que sabemos serem dificultados pela árdua tarefa de proceder às contagens de forma manual. Esses estudos bibliométricos de citações de obras e autores podem ser facilitados pela adoção de critérios mais objetivos de indexação e pelo desenvolvimento de sistemas de levantamento mais amigáveis.

c) As referências de obras gerais não são adequadas para sair da zona de conforto, pois repetem, no mínimo, o equívoco apresentado no item a. Essas obras podem e devem ser utilizadas como fundamentação teórica, mas causa estranhamento que sejam predominantes dentro de uma área específica. 
d) Deve-se sempre ampliar as pesquisas com a produção das práticas do campo nacionais, utilizando com mais frequência as evidências empíricas descobertas pelos pesquisadores de anos anteriores. Não vimos esse procedimento nessa pesquisa, poucos utilizaram mesmo as pesquisas gerais, como a Ação Social das Empresas do Instituto de Pesquisa Econômica Aplicada [IPEA] que tem relatórios desde 2000.

e) A comparação é uma forma de fazer progredir a ciência. A RSE mostra enorme capacidade interdisciplinar. As comparações entre campos exigem mais do pesquisador, mas podem fornecer excelentes insights para desdobramentos e aperfeiçoamentos de várias disciplinas das Ciências Sociais Aplicadas.

Da nossa parte continuaremos a ampliar esta pesquisa para outras bases buscando alcançar o objetivo de um mapa do conhecimento nacional sobre a RSE e aprofundar os estudos sobre a natureza das descobertas reveladas neste artigo.

Artigo recebido em 24.10.2008. Aprovado em 24.04.2009.

\section{REFERENCIAS BIBLIOGRÁFICAS}

Alvarado, U. R. (2002). A lei de Lotka na bibliometria brasileira. Ciência da Informação, 31(2), 1420 .

Amaral, R. M., Freitas, M. P., Souza, L. M. S., Castro, R. O., Coutinho, V. D. G., \& Faria, L. I. L. (2007, setembro). Criação de indicadores sobre a biblioteca digital de teses e dissertações da BCo/UFSCAR através de análise bibliométrica automatizada. Resumos do Seminário Internacional de Bibliotecas Digitais, Campinas, SP, Brasil, 5.

Ashley, P. A. (Coord.). (2003). Ética e responsabilidade social nos negócios. São Paulo: Saraiva.

Baumgartner, H., \& Pieters, R. (2003). The structural influence of marketing journals: a citation analysis of the discipline and its subareas over time. Journal of Marketing, 67(2), 123-139.

Bertero, C. O., Binder, M. P., \& Vasconcelos, F. C. (2003). Estratégia empresarial: a produção científica brasileira entre 1991 e 2002. Revista de Administração de Empresas, 43(4), 48-62.

Bertero, C. O., Caldas, M. P., \& Wood, T., Jr. (Orgs.). (2005). Produção científica em administração no Brasil: o estado da arte. São Paulo: Atlas.

Boltanski, L., \& Chiapello, E. (2002). El nuevo espíritu del capitalismo. (M. P. Colina, Trad.). Madrid: Akal. (Obra original publicada em 1999).

Bordons, M., \& Zulueta, A. (1999). Evaluación de la actividad científica através de indicadores bibliométricos. Revista Española de Cardiologia, 10(52), 790-800.

Boyack, K. W., Wylie, B. N., \& Davidson, G. S. (2002). Domain visualization using vxinsight for science and technology management. Journal of the American Society for Information Science and Technology, 53(9), 764-774.

Caldas, M. P., Tinoco, T., \& Chu, R. A. (2003, setembro). Análise bibliométrica dos artigos de RH publicados no EnANPAD, na década de 1990: um mapeamento a partir das citações dos heróis, endogenias e jactâncias que fizeram a história recente da produção científica da área. Anais do Encontro Nacional da Associação Nacional de Pós-Graduação e Pesquisa em Administração, Atibaia, SP, Brasil, 27. 
Carrol, A. C. (1999). Corporate social responsibility. Business and Society, 3(38), 268-295.

Curado, I. B. (2003, setembro). Responsabilidade legal, responsabilidade social: uma questão de autoridade? Anais do Encontro Nacional da Associação Nacional de Pós-Graduação e Pesquisa em Administração, Atibaia, SP, Brasil, 27.

Donaldson, T., \& Preston, L. E. (1995). The stakeholder theory of the corporation: concepts, evidence, and implications. Academy of Management Review, 20(1), 65-91.

Ferrell, O. C., Fraedrich, J., \& Ferrel, L. (2001). Ética empresarial: dilema, tomada de decisões e casos. (R. Jungmann, Trad.). Rio de Janeiro: Reichmann \& Affonso. (Obra original publicada em 2001).

Freeman, R. E. (2003). Teorias do público interessado. In C. L. Cooper, \& C. Argyris (Orgs.). Dicionário enciclopédico de administração (pp. 1335-1341). (A. Z. Sanvicente, Trad.). São Paulo: Atlas. (Obra original publicada em 1998).

Friedman, M. (1970, September 13). The social responsibility of business to increase its profits. New York Times Magazine, pp. 32-33.

Garfield, D. E. (1973). Essays of an information scientist. Philadelphia: ISI Press.

Garfield, D. E. (1995). Quantitative analysis of the scientific literature and its implication for science policymaking Latin América and the Caribbean. Bulletin of the Pan American Health Organization, 29(1), 87-95.

Gomes, A., \& Moretti, S. L. A. (2007). A responsabilidade e o social: uma discussão sobre o papel das empresas. São Paulo: Saraiva.

Guedes, V. V., \& Borschiver, S. (2005, junho). Bibliometria: uma ferramenta estatística para a gestão da informação e do conhecimento, em sistemas de informação, de comunicação e de avaliação científica e tecnológica. Anais do Encontro Nacional de Ciências da Informação, Salvador, BA, Brasil, 6.

Holbrook, J. A. D. (1992). Why measure science? Science and Public Policy, 19(5), 262-266.

Institute for Scientific Information. (2007). Pesquisa geral no site. Recuperado em 20 setembro, 2007, de http://scientific.thomson.com/isi/

Instituto de Pesquisa Econômica Aplicada. (2000). Ação social das empresas no Brasil: quem são e onde estão. Rio de Janeiro: Autor.

Instituto Ethos de Empresas e Responsabilidade Social. (2001). Guia de elaboração de relatório e balanço anual de responsabilidade social empresarial. São Paulo: Autor.

Jensen, M. C. (2002). Value maximization, stakeholder theory, and the corporate objective function. Business Ethics Quarterly, 12(2), 235-256.

Kreitlon, M. P. (2004, setembro). A ética nas relações entre empresas e sociedade: fundamentos teóricos da responsabilidade social empresarial. Anais do Encontro Nacional da Associação Nacional de Pós-Graduação e Pesquisa em Administração, Curitiba, PR, Brasil, 28.

Leong, S. M. (1989). A citation analysis of the journal of consumer research. Journal of Consumer Research, 15(4), 492-497.

Lipovetsky, G. (2004). Metamorfoses da cultura liberal: ética, mídia e empresa (J. M. Silva, Trad.). Porto Alegre: Sulina. (Obra original publicada em 2002). 
Logsdon, J. M., \& Yuthas, K. (1997). Corporate social performance, stakeholder orientation, and organizational moral development. Journal of Business Ethics, 16(12/13), 1213-1226.

Macke, J., \& Carrion, R. M. (2006, setembro). Programas sociais corporativos: a importância da abordagem transdisciplinar. Anais do Encontro Nacional da Associação Nacional de PósGraduação e Pesquisa em Administração, Salvador, BA, Brasil, 30.

Margolis, J. D., \& Walsh, J. P. (2003). Misery loves companies: rethinking social iniciatives by business. Administrative Science Quarterly, 48(2), 268-305.

McAlister, L. (2005). Unleashing potential. In Marketing renaissance: opportunities and imperatives for improving marketing thought, practice, and infrastructure. Journal of Marketing, 69(4), 1617.

Melo, F. P., Neto, \& Froes, C. (2001). Gestão da responsabilidade social corporativa: o caso brasileiro: da filantropia tradicional à filantropia de alto rendimento e ao empreendedorismo social. Rio de Janeiro: Qualitymark.

Moretti, S. L. A., \& Figueiredo, J. C. (2007, setembro). Análise bibliométrica da produção sobre responsabilidade social das empresas no EnANPAD: evidências de um discurso monológico. Anais do Encontro Nacional da Associação Nacional de Pós-Graduação e Pesquisa em Administração, Rio de janeiro, RJ, Brasil, 31.

Mugnaini, R., Jannuzzi, P., \& Quonian, L. (2004). Indicadores bibliométricos da produção científica brasileira: uma análise a partir da base Pascal. Ciência da Informação, 33(2), 123-131.

Nicholls, P. T. (1989). Bibliometric modeling process and the empirical validity of Lotka's Law. Journal of the American Society for Information Science, 40(6), 379-385.

Pao, M. L. (1989). Concepts of information retrieval. Englewood: Libraries Unlimeted.

Passador, C. S., Canopf, L., \& Passador, J. L. (2005, setembro). Apontamentos sobre a responsabilidade social no EnANPAD: a construção de um conceito? Anais do Encontro Nacional da Associação Nacional de Pós-Graduação e Pesquisa em Administração, Brasília, DF, Brasil, 29.

Pereira, W. A., \& Campos, F. L. A. N. (2006, setembro). Investigação sobre as semelhanças entre os modelos conceituais da responsabilidade social corporativa. Anais do Encontro Nacional da Associação Nacional de Pós-Graduação e Pesquisa em Administração, Salvador, BA, Brasil, 30 .

Price, D. J. D. (1965). Network of scientific paper. Science, 149, 510-515.

Pritchard, A. (1969). A statistical bibliography or bibliometrics? Journal of Documentaries, 25(4), 348-349.

Quonian, L. (1992). Bibliométrie sur des références bibliographiques: méthodologie. In D. Desvals, \& H. Dou (Orgs.). La veille technologique (pp.244-262). Paris: Dumond.

Quonian, L., Tarapanoff, K., Araujo, R. H., Jr., \& Alvares, L. (2001). Inteligência obtida pela aplicação de data mining em base de teses francesas sobre o Brasil. Ciência da Informação, 30(2), 20-28.

Rodrigues, L. C., \& Riccardi, R. (2007). Inteligência competitiva: nos negócios e organizações. Maringá: Unicorpore.

Sampaio, H. C., \& Perin, G. M. (2006). Pesquisa científica na área de marketing: uma revisão histórica. Revista de Administração Contemporânea, 10(2), 179-202. 
Siegel, S. (1975). Estatística não-paramétrica para as ciências do comportamento. São Paulo: McGraw-Hill.

Small, H. (1999). Visualizing science by citation mapping. Journal of the American Society for Information Science, 50(9), 799-813.

Spinak, E. (1998). Indicadores cienciométricos. Ciência da informação, 27(2), 141-148.

Stremersch, S., Vernieers, I., \& Verhoef, P. C. (2007). The quest for citations: drivers of article impact. Journal of Marketing, 71(3), 171-193.

Thompson, J. W. (2002). The death of the scholarly monograph in the humanities? Citation patterns in literary scholarship. Libri, 52, 121-136.

Tyson, K. W. M. (1998). The complete guide to competitive intelligence. Chicago: Kirk Tyson International.

Vanti, N. A. P. (2002). Da bibliometria à webmetria: uma exploração conceitual dos mecanismos utilizados para medir o registro da informação e a difusão do conhecimento. Ciência da Informação, 31(2), 152-162.

Ventura, E. C. F. (2003, setembro). Responsabilidade social das empresas sob a ótica do "novo espírito do capitalismo". Anais do Encontro Nacional da Associação Nacional de PósGraduação e Pesquisa em Administração, Campinas, SP, Brasil, 27.

Vieira, F. G. (2003). Narciso sem espelho: a publicação brasileira de marketing. Revista de Administração de Empresas, 43(1), 81-90.

Weber, M. (2004). A ética protestante e o "espírito" do capitalismo. (J. M. M. Macedo, Trad.). São Paulo: Cia das Letras. (Obra original publicada em 1983).

White, H. D., \& McCain, K. W. (1989). Bibliometrics. Annual Review of Information Science and Technology, 24, 119-186.

Wood, D. J. (1991). Corporate social performance. Academy of Management Review, 16(14), 691-718.

Zhao, L. (2006). How library used e-resources - an analysis of citation in CCQ. Cataloging and Classification Quarterly, 42(1), 117-131. 